\title{
Changes of expression levels of serum cystatin $C$ and soluble vascular endothelial growth factor receptor 1 in the treatment of patients with glomerulus nephritis
}

\author{
XINWEI XU ${ }^{1}$, LILI QIN ${ }^{1}$ and LIPING YAN ${ }^{2}$ \\ ${ }^{1}$ Nephrology Department and ${ }^{2}$ Human Resources Department, \\ Weifang People's Hospital, Weifang, Shandong 261041, P.R. China
}

Received June 6, 2019; Accepted January 20, 2020

DOI: $10.3892 /$ etm.2020.8824

\begin{abstract}
Expression levels and changes of serum cystatin C (C's C) and soluble vascular endothelial growth factor receptor 1 (sVEGFR1) in treatment of patients with glomerulus nephritis (GN) were investigated. The medical records of 88 patients with GN who were diagnosed in Weifang People's Hospital from March 2014 to June 2017 were collected, and their medical records were considered as a study group. The study group was divided into secondary glomerulonephritis (SGN) group (52 cases) and primary glomerulonephritis (PGN) group (36 cases). Physical examination data of 50 healthy volunteers who were examined in the same hospital during the same period were considered as a control group. The correlation between expression of serum C's $\mathrm{C}$ and expression of sVEGFR1 of patients with GN was compared. Expression levels of serum C's $\mathrm{C}$ and sVEGFR 1 of patients before treatment in the study group were higher than those in the control group $(\mathrm{P}<0.05)$. With the extension of the treatment cycle, C's C and sVEGFR1 expression levels in $\mathrm{PGN}$ and $\mathrm{SGN}$ groups reduced gradually $(\mathrm{P}<0.05)$. With the extension of the treatment cycle, the renal function indexes of the study group patients showed a downward trend $(\mathrm{P}<0.05)$. Expression of $\mathrm{C}^{\prime} \mathrm{s} \mathrm{C}$ was positively correlated with urea nitrogen and creatinine $(\mathrm{P}<0.05)$. In conclusion, $\mathrm{C}^{\prime} \mathrm{s} \mathrm{C}$ and sVEGFR1 are highly expressed in the serum of patients with GN. Expression of C's C and sVEGFR1 decrease as patients are treated. C's C and sVEGFR1 can be used as indicators for monitoring the condition of patients with GN. It is worthwhile to promote $\mathrm{C}$ 's $\mathrm{C}$ and sVEGFR1 in clinical practice.
\end{abstract}

Correspondence to: Dr Liping Yan, Human Resources Department, Weifang People's Hospital, 151 Guangwen Street, Kuiwen, Weifang, Shandong 261041, P.R. China

E-mail: peid94@163.com; ylpwf1111@126.com

Key words: glomerulus nephritis, cystatin $\mathrm{C}$, soluble vascular endothelial growth factor receptor 1 , disease monitoring

\section{Introduction}

Glomerulus nephritis (GN) is an autoimmune system disease with complex pathogenesis, diverse pathological classifications and long disease course (1). It can be induced by various causes and can be divided into primary glomerulonephritis (PGN) and secondary glomerulonephritis (SGN) (2). The pathogenic process of GN is divided into several stages: first edema, hematuria or proteinuria can be seen in the body; then, renal function weakens; eventually, patients suffer renal failure (3). At present, the drugs used to treat GN in clinic are few, and the efficacy of some drugs is poor, causing adverse reactions. Moreover, the treatment is expensive (4). Comprehensive preventive measures should be taken in the treatment of GN to control deterioration of renal function, alleviate the symptoms of patients, reduce the incidence of serious complications and improve patients' life quality (5).

Serum cystatin C (C's C) is a non-glycosylated small protein, with 120 amino acid residues, and is a member of cysteine protease inhibitors (6). The production rate of C's C is relatively constant in body fluids and nucleated cells in tissues of the body. C's $\mathrm{C}$ metabolizes and regulates peptides and proteins in cells (7). In the blood circulation of the body, $\mathrm{C}^{\prime} \mathrm{s} \mathrm{C}$ is filtered by glomerular filtration membrane and is reabsorbed by proximal convoluted tubules. The glomerular filtration function directly determines the level of C's $\mathrm{C}$ in the serum $(8,9)$.

Vascular endothelial growth factor (VEGF), a dynamic source of division and growth of endothelial cells, facilitates angiogenesis. VEGF can combine with soluble vascular endothelial growth factor receptor 1 (sVEGFR1) to prevent VEGF membrane receptors from mediating and taking a biological effect. sVEGFR1 and VEGF membrane receptors are competitive, when they are combined with VEGF, the biological activity of sVEGFR1 and VEGF will be regulated negatively $(10,11)$. Studies have shown that when sVEGFR1 combines with VEGF in the blood circulation, the function of VEGF will be regulated. Increasing the level of sVEGFR1 prevents VEGF from facilitating angiogenesis and injuring endothelial cells, which is closely related to kidney diseases (12). 
Studies on the expression of serum C's C and sVEGFR1 in patients with GN and predictive function and evaluation function of serum C's C and sVEGFR1 in diseases are scarce. Therefore, expression levels of serum C's C and sVEGFR1 in the treatment of patients with GN were detected and the monitoring function and significance of serum $\mathrm{C}$ 's $\mathrm{C}$ and sVEGFR1 in GN were investigated in this study, in order to provide specific study data and theoretical basis for clinical treatment of GN.

\section{Patients and methods}

General data. The medical records of 88 patients with GN who were diagnosed in Weifang People's Hospital (Weifang, China) from March 2014 to June 2017 were collected, and their medical records were considered as study group. The study group was divided to SGN group and PGN group. There were 52 patients in SGN group and 36 patients in PGN group. Physical examination data of 50 healthy volunteers who were examined in Weifang People's Hospital during the same period were considered as the control group. Among those patients, there were 66 males and 72 females, with an average age of $45.0 \pm 15.7$ years and a BMI of $21.0 \pm 3.6$. There was no significant difference in some data of the patients in two groups, including gender, age and BMI $(\mathrm{P}>0.05)$. There were significant differences in hypertension, edema and some other aspects $(\mathrm{P}<0.05)$ (Table I).

Inclusion and exclusion criteria. Inclusion criteria: Patients who were diagnosed with GN by histopathology were included; Serum creatinine (SCr) $<350 \mathrm{~mol} / \mathrm{l}$, and blood urea nitrogen $(\mathrm{BUN})<17 \mathrm{mmol} / \mathrm{l}$; urine $\mathrm{RBC}$ was increased and showed tubular shape (13).

Exclusion criteria: i) patients with malignant tumors; ii) patients complicated with solid lesions of other organs; iii) patients complicated with blood diseases and infection; iv) patients with cognitive disorder or communication disorder; v) patients with poor compliance.

All the patients and their family members agreed to participate in the experiment, and an informed consent form was signed. This experiment was approved by the Medical Ethics Committee of Weifang People's Hospital.

Reagents and materials. The serum separation centrifuge was purchased from Zhengzhou Zhishi Changyun Technology Co., Ltd. C's C was was detected by automatic biochemical analyzer BS-600 (Shenzhen Mindray Biomedical Electronics Co.,Ltd.); C's C test kits (Shenzhen ziker Biological Technology Co., Ltd., ZK-H068); sVEGFR1 kits were from R\&D Systems (ML-ELISA-0073).

Experiment methods. The patients were treated for more than 3 cycles. The specific therapeutic regimen was as follows: the patients took valsartan dispersible tablets once a day, and the dosage was $80 \mathrm{mg}$ per time. Alprostadil injection $(10 \mu \mathrm{g})$ was mixed with sodium chloride solution with a concentration of $0.9 \%$, then the patients were injected with the mixture once a day, the dosage was $100 \mathrm{ml}$ per time. Fasting venous blood $(5 \mathrm{ml})$ of the patients and the healthy volunteers was collected in the morning, then the serum was separated from the blood by serum separation centrifuge at a speed of $1,500 \mathrm{x} \mathrm{g}$ at $4^{\circ} \mathrm{C}$ for $10 \mathrm{~min}$, and the serum was reserved. Serum C's $C$ of the patients was detected by immunoturbidimetry. The expression level of sVEGFR1 in the serum was detected by enzyme-linked immunosorbent assay (ELISA). The procedures were carried out strictly according to the instructions of the kits.

Observation indicators. i) The expression levels of C's C and sVEGFR1 in serum of the patients in the study group and the control group were compared before treatment. ii) The changes of expression levels of serum C's C of the patients in PGN group and SGN group were compared before they were treated, after one cycle of treatment, after three cycles of treatment and after discharged from the hospital. iii) The changes of expression levels of sVEGFR1 of the patients in PGN group and SGN group were compared before they were treated, after one cycle of treatment, after three cycles of treatment and after discharged from the hospital. iv) The correlation between the expression of C's $\mathrm{C}$ and the expression of sVEGFR1 in GN was analyzed by correlation analysis.

Statistical analysis. In this study, SPSS 19.0 (Bizinsight (Beijing) Information Technology Co., Ltd.) was used to statistically analyze the experimental data. The count data were analyzed by $\chi^{2}$ test. The measurement data were expressed in the form of mean value \pm standard deviation. t-test was used in comparison of data between two groups. Bonferroni test was the post hoc test. Repeated measures analysis of variance was used in comparison of the data between two groups at different time points in the treatment of patients. Pearson analysis was used to carry out correlation analysis of the data. The illustrations of this experiment were drawn by GraphPad Prism 8. P $<0.05$ indicates statistically significant difference.

\section{Results}

Comparison of expression levels of C's $C$ and sVEGFRI of the patients in the study and the control groups. The expression levels of C's C and sVEGFR1 of the patients in the study group and the subjects in the control group were compared before treatment. The expression level of C's C of the patients in the study group was significantly higher than that in control group. The expression level of sVEGFR1 of the patients in study group was significantly higher than that in the control group. Differences in expression levels of C's C and sVEGFR1 of the patients in the two groups were statistically significant $(\mathrm{P}<0.05)$ (Table II).

Comparison of the changes of expression level of $C^{\prime} s C$ of the patients in PGN and SGN groups before treatment, one cycle after treatment, three cycles after treatment and after discharge. The expression level of C's $\mathrm{C}$ of the patients in PGN group and SGN group was compared before treatment, after one cycle of treatment, after three cycles of treatment, and after discharge from hospital. It was found that the expression level of C's C of the patients in PGN group and SGN group decreased gradually after one cycle of treatment. 
Table I. General data of the patients in the study group and the control group [n (\%)].

\begin{tabular}{|c|c|c|c|c|}
\hline Factors & Study group $(\mathrm{n}=88)$ & Control group $(\mathrm{n}=50)$ & $\chi^{2} / \mathrm{t}$ & P-value \\
\hline Sex & & & 0.105 & 0.746 \\
\hline Male & $43(48.86)$ & $23(46.00)$ & & \\
\hline Female & $45(51.14)$ & $27(54.00)$ & & \\
\hline Age (years) & & & 0.001 & 0.975 \\
\hline$\leq 45$ & $46(52.27)$ & $26(52.00)$ & & \\
\hline$>45$ & $42(47.73)$ & $24(48.00)$ & & \\
\hline $\operatorname{BMI}\left(\mathrm{kg} / \mathrm{m}^{2}\right)$ & & & 0.178 & 0.673 \\
\hline$\leq 21$ & $42(47.73)$ & $22(44.00)$ & & \\
\hline$>21$ & $46(52.27)$ & $28(56.00)$ & & \\
\hline Hypertension & & & 48.391 & $<0.001$ \\
\hline Yes & $63(71.59)$ & $5(10.00)$ & & \\
\hline No & $25(28.41)$ & $45(90.00)$ & & \\
\hline Edema & & & 63.971 & $<0.001$ \\
\hline Yes & $62(70.45)$ & 0 & & \\
\hline No & $26(29.55)$ & $50(100.00)$ & & \\
\hline Hematuresis & & & 60.311 & $<0.001$ \\
\hline Yes & $60(68.18)$ & 0 & & \\
\hline No & $28(31.82)$ & $50(100.00)$ & & \\
\hline Proteinuria & & & 76.171 & $<0.001$ \\
\hline Yes & $68(77.27)$ & 0 & & \\
\hline No & $20(22.73)$ & $50(100.00)$ & & \\
\hline Urea nitrogen $(\mathrm{mmol} / \mathrm{l})$ & $14.34 \pm 1.23$ & $5.65 \pm 0.83$ & 44.500 & $<0.001$ \\
\hline Creatinine $(\mu \mathrm{mol} / \mathrm{l})$ & $293.43 \pm 32.32$ & $81.34 \pm 11.25$ & 44.820 & $<0.001$ \\
\hline
\end{tabular}

Table II. Comparison of expression levels of C's C and sVEGFR1 in the two groups.

\begin{tabular}{lccr}
\hline Groups & Study group $(\mathrm{n}=88)$ & Control group $(\mathrm{n}=50)$ & $\mathrm{t}$ \\
\hline C'sC $(\mathrm{mg} / \mathrm{l})$ & $2.43 \pm 0.45$ & $0.83 \pm 0.18$ & 24.040 \\
sVEGFR1 $(\mu \mathrm{g} / \mathrm{l})$ & $30.42 \pm 7.42$ & $7.34 \pm 1.54$ & 21.700 \\
\hline
\end{tabular}

C's C, serum cystatin C; sVEGFR1, soluble vascular endothelial growth factor receptor 1.

The expression level of C's C of the patients in PGN group and SGN group after one cycle of treatment was lower than that before they were treated $(\mathrm{P}<0.05)$. The expression level of C's $\mathrm{C}$ of the patients in PGN group and SGN group decreased gradually after three cycles of treatment. The expression level of C's C of the patients in PGN group and SGN group after three cycles of treatment was lower than that after one cycle of treatment $(\mathrm{P}<0.05)$. The expression level of C's $\mathrm{C}$ of the patients in PGN group and SGN group decreased gradually after discharge from hospital. The expression level of C's C of the patients in PGN group and SGN group after discharged from the hospital was lower than that after three cycles of treatment $(\mathrm{P}<0.05)$. There was no significant difference between the expression level of $C^{\prime}$ 's $C$ of the patients in PGN group and that in SGN group $(\mathrm{P}>0.05)$ (Table III and Fig. 1).
Comparison of the changes of expression level of SVEGFRI of the patients in PGN and SGN groups before treatment, one cycle after treatment, in three cycles after treatment and after discharge. The expression level of sVEGFR1 of the patients in PGN group and SGN group was compared before they were treated, after a cycle of treatment, after three cycles of treatment, and after they discharged from the hospital. It was found that the expression level of sVEGFR1 of the patients in PGN group and SGN group decreased gradually after a cycle of treatment. The expression level of sVEGFR1 of the patients in PGN group and SGN group after a cycle of treatment was lower than that before they were treated $(\mathrm{P}<0.05)$. The expression level of sVEGFR1 of the patients in PGN group and SGN group decreased gradually after three cycles of treatment. The expression level of sVEGFR1 of the patients in PGN group and SGN group after 
Table III. Comparative analysis of the expression level of C's C of the patients in PGN group and SGN group before and after treatment.

\begin{tabular}{lcccccr}
\hline & $\begin{array}{c}\text { Before } \\
\text { patients } \\
\text { were treated }\end{array}$ & $\begin{array}{c}\text { In one cycle } \\
\text { after patients } \\
\text { were treated }\end{array}$ & $\begin{array}{c}\text { In three cycles } \\
\text { after patients } \\
\text { were treated }\end{array}$ & $\begin{array}{c}\text { After patients } \\
\text { were discharged }\end{array}$ & F-value & P-value \\
\hline SGN group (n=52) & $2.42 \pm 0.42$ & $2.11 \pm 0.34^{\mathrm{a}}$ & $1.62 \pm 0.30^{\mathrm{a}, \mathrm{b}}$ & $0.45 \pm 0.22^{\mathrm{a}-\mathrm{c}}$ & 0.842 & $<0.001$ \\
PGN group (n=36) & $2.43 \pm 0.45$ & $2.12 \pm 0.42^{\mathrm{a}}$ & $1.64 \pm 0.35^{\mathrm{a}, \mathrm{b}}$ & $0.42 \pm 0.24^{\mathrm{a}-\mathrm{c}}$ & 0.812 & $<0.001$ \\
t-test & 0.107 & 0.123 & 0.287 & 0.606 & & \\
P-value & 0.915 & 0.902 & 0.775 & 0.546 & & \\
\hline
\end{tabular}

${ }^{\mathrm{a}} \mathrm{P}<0.05$, compared with before treatment in the same group; ${ }^{\mathrm{b}} \mathrm{P}<0.05$ compared with one cycle of treatment in the same group; ${ }^{\mathrm{C}}<0.05$ compared with three cycles of treatment in the same group. C's C, serum cystatin C; PGN, primary glomerulonephritis; SGN, secondary glomerulonephritis.

Table IV. Comparative analysis of the expression level of sVEGFR1 of the patients in PGN group and SGN group before and after treatment.

\begin{tabular}{lccccrl}
\hline & $\begin{array}{c}\text { Before the } \\
\text { patients } \\
\text { were treated }\end{array}$ & $\begin{array}{c}\text { In one cycle } \\
\text { after the patients } \\
\text { were treated }\end{array}$ & $\begin{array}{c}\text { In three cycles } \\
\text { after the patients } \\
\text { were treated }\end{array}$ & $\begin{array}{c}\text { After the patients } \\
\text { were discharged }\end{array}$ & F-value & P-value \\
\hline SGN group (n=52) & $30.51 \pm 7.13$ & $26.43 \pm 6.46^{\mathrm{a}}$ & $21.42 \pm 5.25^{\mathrm{a}, \mathrm{b}}$ & $17.34 \pm 5.13^{\mathrm{a}-\mathrm{c}}$ & 0.409 & $<0.001$ \\
PGN group (n=36) & $30.42 \pm 7.42$ & $26.32 \pm 6.42^{\mathrm{a}}$ & $21.53 \pm 5.63^{\mathrm{a}, \mathrm{b}}$ & $17.45 \pm 5.26^{\mathrm{a}-\mathrm{c}}$ & 0.387 & $<0.001$ \\
t-test & 0.057 & 0.079 & 0.094 & 0.098 & & \\
P-value & 0.955 & 0.937 & 0.926 & 0.922 & & \\
\hline
\end{tabular}

${ }^{\mathrm{a}} \mathrm{P}<0.05$, compared with before treatment in the same group; ${ }^{\mathrm{b}} \mathrm{P}<0.05$ compared with a cycle of treatment in the same group; ${ }^{\mathrm{C}} \mathrm{P}<0.05$ compared with three cycles of treatment in the same group. sVEGFR1, soluble vascular endothelial growth factor receptor 1; PGN, primary glomerulonephritis; SGN, secondary glomerulonephritis.

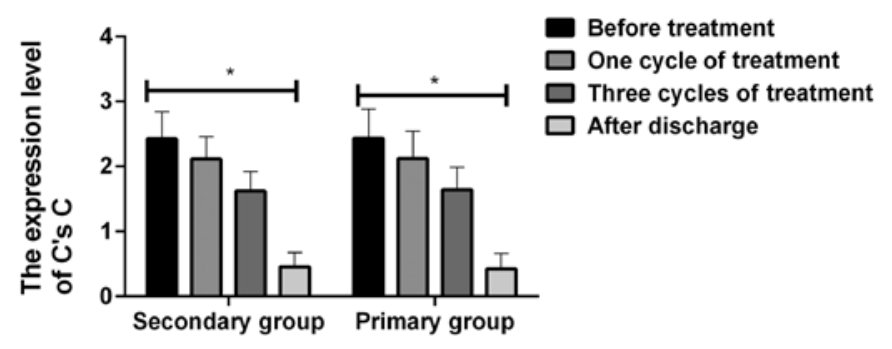

Figure 1. Comparison of the expression level of C's C of the patients in PGN group and SGN group before treatment, in one cycle after treatment, in three cycles after treatment and after they were discharged from hospital. The result of immunoturbidimetry showed that the expression leve of C's C of the patients in PGN group and SGN group decreased gradually in one cycle after treatment. The expression level of $\mathrm{C}^{\prime} \mathrm{s} \mathrm{C}$ of the patients in PGN group and SGN group one cycle after treatment was lower than that before treatment $(\mathrm{P}<0.05)$. Expression level of $\mathrm{C}^{\prime} \mathrm{s} \mathrm{C}$ of the patients in PGN group and SGN group decreased gradually in three cycles after treatment. Expression level of C's C of the patients in PGN group and SGN group in three cycles after treatment was lower than that in one cycle after treatment $(\mathrm{P}<0.05)$. Expression level of $\mathrm{C}$ 's $\mathrm{C}$ of the patients in $\mathrm{PGN}$ group and SGN group decreased gradually after they were discharged from hospital. Expression level of C's C of the patients in PGN group and SGN group after discharge was lower than that in three cycles after they were treated $(\mathrm{P}<0.05)$. There was no significant difference between the expression level of C's $\mathrm{C}$ of the patients in PGN group and that in SGN group $(\mathrm{P}>0.05)$. " $\mathrm{P}<0.05$. C's $\mathrm{C}$, serum cystatin C; PGN, primary glomerulonephritis; SGN, secondary glomerulonephritis.

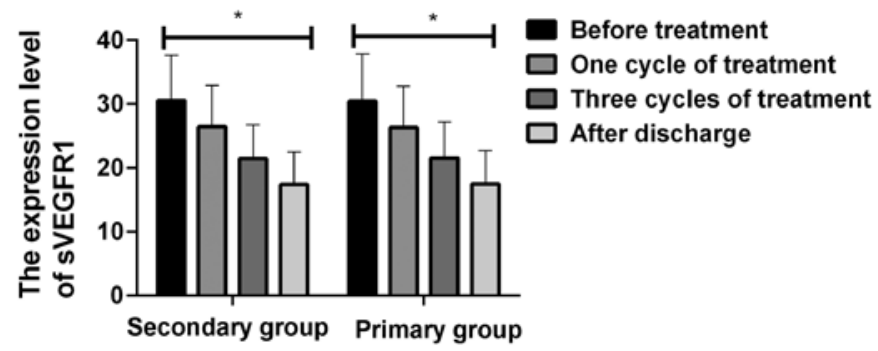

Figure 2. Comparison of the expression level of sVEGFR1 of the patients in PGN group and SGN group before treatment, in one cycle after treatment, in three cycles after treatment and after they were discharged from hospital. The result of ELISA showed that the expression level of sVEGFR1 of the patients in PGN group and SGN group decreased gradually in one cycle after treatment. Expression level of sVEGFR1 of the patients in PGN group and SGN group one cycle after treatment was lower than that before treatment $(\mathrm{P}<0.05)$. Expression level of sVEGFR1 of the patients in PGN group and SGN group decreased gradually in three cycles after treatment. Expression level of sVEGFR1 of the patients in PGN group and SGN group in three cycles after they were treated was lower than that in one cycle after they were treated $(\mathrm{P}<0.05)$. Expression level of sVEGFR1 of the patients in PGN group and SGN group decreased gradually after discharge from hospital. Expression level of sVEGFR1 of the patients in PGN group and SGN group after discharge was lower than that in three cycles after they were treated $(\mathrm{P}<0.05)$. There was no significant difference between the expression level of sVEGFR1 of the patients in PGN group and that in $\mathrm{SGN}$ group $(\mathrm{P}>0.05)$. ${ }^{*} \mathrm{P}<0.05$. sVEGFR1, soluble vascular endothelial growth factor receptor 1; PGN, primary glomerulonephritis; SGN, secondary glomerulonephritis. 
Table V. Changes in renal function of the study group in different periods of treatment.

\begin{tabular}{lcccccc}
\hline & $\begin{array}{c}\text { Before } \\
\text { patients } \\
\text { were treated }\end{array}$ & $\begin{array}{c}\text { One cycle } \\
\text { after patients } \\
\text { were treated }\end{array}$ & $\begin{array}{c}\text { Three cycles } \\
\text { after patients } \\
\text { were treated }\end{array}$ & $\begin{array}{c}\text { After patients } \\
\text { were discharged }\end{array}$ & F-value & P-value \\
\hline Urea nitrogen (mmol/l) & $14.34 \pm 1.23$ & $12.42 \pm 1.14^{\mathrm{a}}$ & $10.41 \pm 1.08^{\mathrm{a}, \mathrm{b}}$ & $7.65 \pm 0.84^{\mathrm{a}-\mathrm{c}}$ & 615.500 & $<0.001$ \\
Creatinine (umol/l) & $293.43 \pm 32.32$ & $241.52 \pm 31.02^{\mathrm{a}}$ & $174.41 \pm 30.73^{\mathrm{a}, \mathrm{b}}$ & $161.41 \pm 28.26^{\mathrm{a}-\mathrm{c}}$ & 440.900 & $<0.001$ \\
\hline
\end{tabular}

${ }^{\mathrm{a}} \mathrm{P}<0.05$, compared with before treatment in the same group; ${ }^{\mathrm{b}} \mathrm{P}<0.05$ compared with one cycle of treatment in the same group; ${ }^{\mathrm{c}} \mathrm{P}<0.05$ compared with three cycles of treatment in the same group.
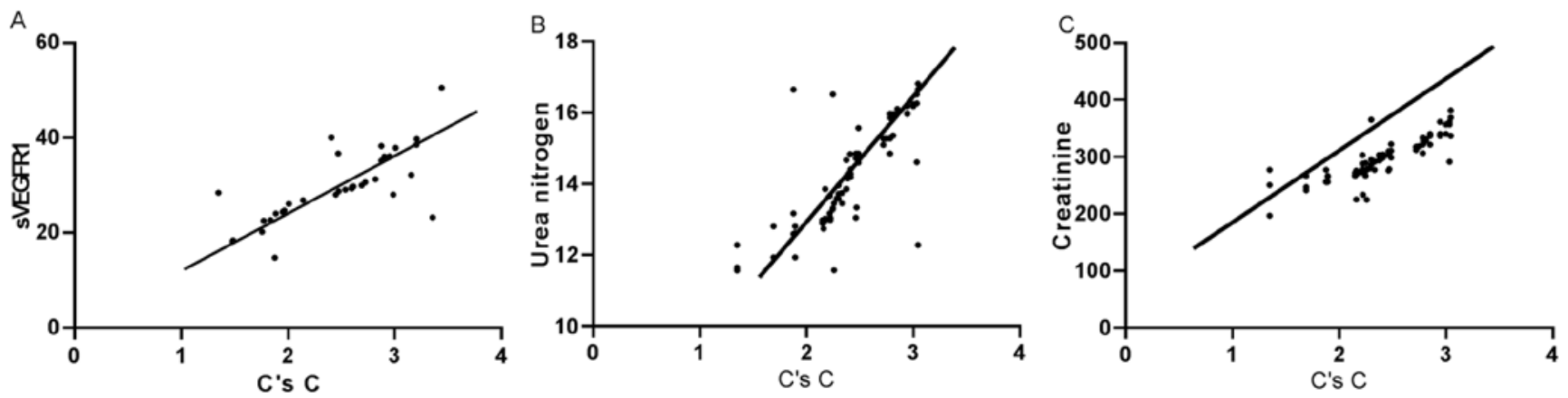

Figure 3. Correlation analysis of expression of C's C and sVEGFR1 in GN, the renal function indexes. (A) According to Spearman's correlation analysis, the expression of C's $\mathrm{C}$ and sVEGFR1 was positively correlated in $\mathrm{GN}(\mathrm{r}=0.740, \mathrm{P}<0.001)$. (B) C's $\mathrm{C}$ expression was positively correlated with urea nitrogen $(\mathrm{r}=0.797, \mathrm{P}<0.001)$. (C) C's C expression was positively correlated with creatinine $(\mathrm{r}=0.841, \mathrm{P}<0.001)$. C's $\mathrm{C}$, serum cystatin $\mathrm{C}$; sVEGFR1, soluble vascular endothelial growth factor receptor 1; GN, glomerulus nephritis; PGN, primary glomerulonephritis; SGN, secondary glomerulonephritis.

three cycles of treatment was lower than that after one cycle of treatment $(\mathrm{P}<0.05)$. The expression level of sVEGFR1 of the patients in PGN group and SGN group decreased gradually after discharge from the hospital. The expression level of sVEGFR1 of the patients in PGN group and SGN group after discharge from hospital was lower than that after three cycles of treatment $(\mathrm{P}<0.05)$. There was no significant difference between the expression level of sVEGFR1 of the patients in PGN group and that in SGN group ( $\mathrm{P}>0.05)$ (Table IV and Fig. 2).

Changes in renal function in different periods of treatment. The results showed that with the increase of the treatment cycle, the renal function indexes of patients in the study group showed a downward trend $(\mathrm{P}<0.05)($ Table $\mathrm{V})$.

Correlation analysis of expression of C's $C$ and SVEGFR1 in $G N$ and the renal function indexes. According to Spearman's correlation analysis, the expression of C's C and sVEGFR1 was positively correlated in $\mathrm{GN}(\mathrm{r}=0.740, \mathrm{P}<0.001)$. Due to the positive correlation between the expression of C's C and sVEGFR1, the association between the expression of $\mathrm{C}^{\prime} \mathrm{s} \mathrm{C}$ and urea nitrogen, creatinine were analyzed. Spearman's correlation results showed that C's $\mathrm{C}$ expression was positively correlated with urea nitrogen ( $r=0.797, \mathrm{P}<0.001)$, and $\mathrm{C}$ 's $\mathrm{C}$ expression was positively correlated with creatinine $(\mathrm{r}=0.841, \mathrm{P}<0.001)$ (Fig. 3).

\section{Discussion}

At present, it is believed that GN can lead to end-stage renal diseases and its pathological changes are complex. For many
GN diseases, cellular immunity plays a decisive role, and humoral immunity is also clearly expressed during the pathogenic process of GN diseases (14). In addition, the disorder of blood coagulation mechanism and the change of blood rheology have an effect on the occurrence and progression of glomerular diseases (15). In clinic, the pathogenesis and treatment of GN are known, the drugs used to treat GN are immunosuppressive agents and glucocorticoid, but the efficacy of many glomerular diseases is still unsatisfactory $(16,17)$. In order to evaluate the changes of patients' condition in the occurrence and progression of GN disease and improve clinical symptoms of the patients, and the cure rate, the expression of C's C and sVEGFR1 in GN was investigated. $C$ 's $\mathrm{C}$ is an independent endogenous marker, only filtered and metabolized by kidneys. C's C is important in the early diagnosis of renal injury and is highly recognized for evaluating glomerular function in clinic $(18,19)$. sVEGFR1 is produced in endothelial cells, monocytes and placenta. As a soluble receptor of VEGF, it negatively regulates the activity of VEGF (20). When sVEGFR1 is combined with VEGF, the signal transduction acts on angiogenesis, when this process is disrupted, a series of pathological conditions will occur, such as the deterioration of renal function, which is caused by injury of vascular endothelial cell function (21).

In this study, the expression levels of C's C and sVEGFR1 of the patients in the study group and the control group were compared. It was found that the expression levels of C's C and sVEGFR1 of the patients in the study group were significantly higher than those in the control group. The results indicated that the expression levels of C's C and sVEGFR1 were diagnostic markers in the occurrence and progression of glomerular 
nephropathy. Some studies reported that the occurrence and progression of GN diseases are related to cellular immunity, serum free radicals, and coagulation mechanism, and that the occurrence and development of GN diseases are closely related to cellular immunity (22). C's C is a cysteine protease inhibitor that can be used to detect the filtration rate of glomeruli in kidney injury. The practical method to detect C's C is immunoassay (23). The signal transduction of sVEGFR1 improves hematopoietic function, proliferative function, differentiation function and immune function of monocytes and macrophages of hematopoietic stem cells (HSC) (24). The changes of cellular immunity exist in the expression of C's C and sVEGFR1, which indirectly confirms the conclusion of this study. To investigate the specific changes of the expression levels of C's C and sVEGFR1 in GN, the changes of expression levels of C's C and sVEGFR1 were investigated before and after treatment of patients. The results showed that expression levels of serum C's C and sVEGFR1 of the patients in PGN group and SGN group decreased gradually after a cycle of treatment. Expression levels of serum C's C and sVEGFR1 of the patients in PGN group and SGN group after a cycle of treatment were lower than those before treatment $(\mathrm{P}<0.05)$. Expression levels of serum C's $\mathrm{C}$ and sVEGFR1 of the patients in PGN group and SGN group decreased gradually after three cycles of treatment. Expression levels of serum C's C and sVEGFR1 of the patients in PGN group and SGN group after three cycles of treatment were lower than those after a cycle of treatment $(\mathrm{P}<0.05)$. Expression levels of serum C's $\mathrm{C}$ and sVEGFR1 of the patients in PGN group and SGN group decreased gradually after they discharged. Expression levels of serum C's $\mathrm{C}$ and sVEGFR1 of the patients in PGN group and SGN group after discharged from hospital were lower than those after three cycles of treatment $(\mathrm{P}<0.05)$. Previous studies have shown that the pathogenesis of secondary glomerular diseases and primary glomerular diseases is completely different, while their clinical manifestations and pathological changes are similar (25). Previous studies have demonstrated that C's C can be expressed effectively in the occurrence and progression of kidney diseases. With the deterioration of pathological conditions, the filtration rate of glomeruli decreases and the expression level of C's C increases (26). When the glomerular filtration barrier is injured and the repair of endothelial cells is blocked, the biological effects of VEGF will be inhibited and respond to the expression of sVEGFR1 negatively (27). In this study, the changes of expression of C's C and sVEGFR1 were investigated before and after treatment. The result showed that the expression levels of C's C and sVEGFR1 decreased with the continuous treatment of the patients. This result indicates that the expression of C's C and sVEGFR1 can be used as an indicator for monitoring the condition of patients with GN when they are treated. The correlation between expression of C's C and expression of sVEGFR1 was analyzed. The result showed that the expression of C's $\mathrm{C}$ and sVEGFR1 was positively correlated in GN. This result suggests that C's $\mathrm{C}$ and sVEGFR1 are associated with the progression of GN. Some studies (28) demonstrated that endogenous creatinine clearance was an important indicator to evaluate the filtration rate of glomeruli. Endogenous creatinine clearance and expression of C's C are related to the pathogenic process of GN. At present, studies on sVEGFR1 and the correlation between C's C and
sVEGFR1 are few, so the specific mechanism of C's $\mathrm{C}$ and sVEGFR1 needs further investigation.

In conclusion, $\mathrm{C}$ 's $\mathrm{C}$ and sVEGFR1 are highly expressed in serum of GN patients. As patients are treated, the expression levels of C's C and sVEGFR1 decrease. C's C and sVEGFR1 play important roles in monitoring the condition of GN patients during treatment.

\section{Acknowledgements}

Not applicable.

\section{Funding}

No funding was received.

\section{Availability of data and materials}

The datasets used and/or analyzed during the current study are available from the corresponding author on reasonable request.

\section{Authors' contributions}

XX analyzed and interpreted the patient general data. LQ performed ELISA. LY was responsible for analysis of the observation indicators. XX wrote the manuscript. All authors read and approved the final manuscript.

\section{Ethics approval and consent to participate}

The study was approved by the Ethics Committee of Weifang People's Hospital (Weifang, China). Patients who participated in this research had complete clinical data. Signed informed consents were obtained from the patients and/or the guardians.

\section{Patient consent for publication}

Not applicable.

\section{Competing interests}

The authors declare that they have no competing interests.

\section{References}

1. Zhao W, Ma Y, Wang M, Shi C, Sun J, Chu K and Liu C: Expression of Foxp3 in renal tissue of patients with HBV-associated glomerulonephritis and their clinical and pathological characteristics. Exp Ther Med 14: 4928-4934, 2017.

2. Agrawal S,Zaritsky JJ,Fornoni A and Smoyer WE: Dyslipidaemia in nephrotic syndrome: Mechanisms and treatment. Nat Rev Nephrol 14: 57-70, 2018

3. Nakagawa N, Hasebe N, Hattori M, Nagata M, Yokoyama H, Sato H, Sugiyama H, Shimizu A, Isaka Y, Maruyama S, et al: Clinical features and pathogenesis of membranoproliferative glomerulonephritis: A nationwide analysis of the Japan renal biopsy registry from 2007 to 2015. Clin Exp Nephrol 22: 797-807, 2018.

4. Komatsu H, Fujimoto S, Yoshikawa N, Kitamura H, Sugiyama H and Yokoyama H: Clinical manifestations of Henoch-Schönlein purpura nephritis and IgA nephropathy: Comparative analysis of data from the Japan Renal Biopsy Registry (J-RBR). Clin Exp Nephrol 20: 552-560, 2016. 
5. Gumber R, Cohen JB, Palmer MB, Kobrin SM, Vogl DT, Wasserstein AG, Nasta SD, Bleicher MB, Bloom RD, Dember L, et al: A clone-directed approach may improve diagnosis and treatment of proliferative glomerulonephritis with monoclonal immunoglobulin deposits. Kidney Int 94: 199-205, 2018.

6. Pottel H, Delanaye P, Schaeffner E, Dubourg L, Eriksen BO, Melsom T, Lamb EJ, Rule AD, Turner ST, Glassock RJ, et al Estimating glomerular filtration rate for the full age spectrum from serum creatinine and cystatin C. Nephrol Dial Transplant 32: 497-507, 2017.

7. Pottel H, Dubourg L, Schaeffner E, Eriksen BO, Melsom T, Lamb EJ, Rule AD, Turner ST, Glassock RJ, De Souza V, et al: The diagnostic value of rescaled renal biomarkers serum creatinine and serum cystatin $\mathrm{C}$ and their relation with measured glomerular filtration rate. Clin Chim Acta 471: 164-170, 2017.

8. Serezlija E, Serdarevic N and Begic L: The estimation of glomerular filtration rate based on the serum cystatin $\mathrm{C}$ and creatinine values. Clin Lab 63: 1099-1106, 2017.

9. Pottel H, Dubourg L, Schaeffner E, Eriksen BO, Melsom T, Lamb EJ, Rule AD, Turner ST, Glassock RJ, De Souza V, et al: Data on the relation between renal biomarkers and measured glomerular filtration rate. Data Brief 14: 763-772, 2017.

10. Trachtman H, Gipson DS, Somers M, Spino C, Adler S, Holzman L, Kopp JB, Sedor J, Overfield S, Elegbe A, et al Randomized clinical trial design to assess abatacept in resistant nephrotic syndrome. Kidney Int Rep 3: 115-121, 2017.

11. Abramowicz D, Oberbauer R, Heemann U, Viklicky O, Peruzzi L, Mariat C, Crespo M, Budde K and Oniscu GC: Recent advances in kidney transplantation: A viewpoint from the Descartes advisory board. Nephrol Dial Transplant 33: 1699-1707, 2018.

12. Bus P, Scharpfenecker M, Van Der Wilk P, Wolterbeek R, Bruijn JA and Baelde HJ: The VEGF-A inhibitor sFLT-1 improves renal function by reducing endothelial activation and inflammation in a mouse model of type 1 diabetes. Diabetologia 60 : 1813-1821, 2017

13. Atkinson JM, Pullen N, Da Silva-Lodge M, Williams L and Johnson TS: Inhibition of thrombin-activated fibrinolysis inhibitor increases survival in experimental kidney fibrosis. J Am Soc Nephrol 26: 1925-1937, 2015

14. Peng T, Xie T, Liu L, Zhen J and Yang X: Analysis of clinical features and pathology of serum HBsAg positive glomerulonephritis. J Med Virol 90: 612-615, 2018.

15. Güran T: Latest insights on the etiology and management of primary adrenal insufficiency in children. J Clin Res Pediatr Endocrinol 9 (Suppl 2): 9-22, 2017.

16. Leon J, Pérez-Sáez MJ, Uffing A, Murakami N, Watanabe A, Cureton P, Kenyon V, Keating L, Yee K, Fernandes Satiro CA et al: Effect of combined gluten-free, dairy-free diet in children with steroid-resistant nephrotic syndrome: An open pilot trial. Kidney Int Rep 3: 851-860, 2018.

17. Krhač M and Lovrenčić MV: Update on biomarkers of glycemic control. World J Diabetes 10: 1-15, 2019.
18. Leem AY, Park MS, Park BH, Jung WJ, Chung KS, Kim SY, Kim EY, Jung JY, Kang YA, Kim YS, et al: Value of serum cystatin $\mathrm{C}$ measurement in the diagnosis of sepsis-induced kidney injury and prediction of renal function recovery. Yonsei Med J 58: 604-612, 2017.

19. De Vriese AS, Sethi S, Nath KA, Glassock RJ and Fervenza FC: Differentiating primary, genetic, and secondary FSGS in adults: A clinicopathologic approach. J Am Soc Nephrol 29: 759-774, 2018.

20. Matsui M, Takeda Y, Uemura S, Matsumoto T, Seno A, Onoue K, Tsushima H, Morimoto K, Soeda T, Okayama S, et al: Suppressed soluble Fms-like tyrosine kinase-1 production aggravates atherosclerosis in chronic kidney disease. Kidney Int 85: 393-403, 2014

21. Li C, Liu B, Dai Z and Tao Y: Knockdown of VEGF receptor-1 (VEGFR-1) impairs macrophage infiltration, angiogenesis and growth of clear cell renal cell carcinoma (CRCC). Cancer Biol Ther 12: 872-880, 2011.

22. Lewis EJ: The enigma of cellular immunity in glomerulonephritis: Lupus nephritis is not necessarily a prototype of immune complex-mediated glomerulonephritis. Nephron Physiol 112: 6-10, 2009.

23. Lin H, Li L, Lei C, Xu X, Nie Z, Guo M, Huang Y and Yao S: Immune-independent and label-free fluorescent assay for cystatin $\mathrm{C}$ detection based on protein-stabilized Au nanoclusters. Biosens Bioelectron 41: 256-261, 2013.

24. Murakami M, Iwai S, Hirasuka S, Buron F, de Souza VC, Rimmelé T, Thaunat O, Badet L, Morelon E, et al: VEGFR-1 (Flt-1) tyrosine kinase signaling enhances hematopoiesis, proliferation/differentiation and immunity of monocyte/macrophage from bone marrow hematopoietic stem cells, and promotes rheumatoid arthritis. Transpl Int 32: 75-83, 2018.

25. Yang Y, Zhang Z, Zhuo L, Chen DP and Li WG: The spectrum of biopsy-proven glomerular disease in China: A systematic review. Chin Med J (Engl) 131: 731-735, 2018

26. Shen ZX, Liu Y, Liu M, Zheng H, Wu XJ and Shen C: Evaluation and application of estimation of glomerular filtration rate based on serum creatinine and cystatin $C$ in renal function staging] Zhonghua Liu Xing Bing Xue Za Zhi 38: 1557-1562, 2017 (In Chinese).

27. Satchell SC, Anderson KL and Mathieson PW: Angiopoietin 1 and vascular endothelial growth factor modulate human glomerular endothelial cell barrier properties. J Am Soc Nephrol 15: 566-574, 2004

28. Nix DE, Mayersohn M and Erstad BL: Should estimates of glomerular filtration rate and creatinine clearance be indexed to body surface area for drug dosing? Am J Health Syst Pharm 74: 1814-1819, 2017.

This work is licensed under a Creative Commons Attribution-NonCommercial-NoDerivatives 4.0 International (CC BY-NC-ND 4.0) License. 